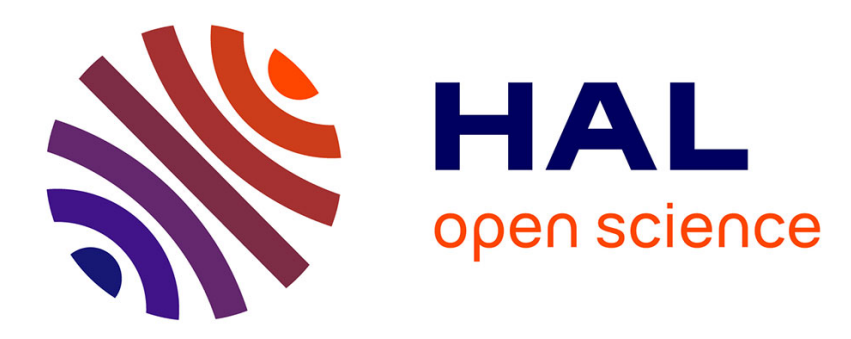

\title{
Kinetic energy released in the vibrational autodetachment of sulfur hexafluoride anion
}

Bruno Concina, Guillaume Montagne, Serge Martin, C. Bordas

\section{To cite this version:}

Bruno Concina, Guillaume Montagne, Serge Martin, C. Bordas. Kinetic energy released in the vibrational autodetachment of sulfur hexafluoride anion. Journal of Chemical Physics, 2021, 154 (23), pp.234306. 10.1063/5.0054199 . hal-03340128

\section{HAL Id: hal-03340128 \\ https://hal.science/hal-03340128}

Submitted on 27 Oct 2021

HAL is a multi-disciplinary open access archive for the deposit and dissemination of scientific research documents, whether they are published or not. The documents may come from teaching and research institutions in France or abroad, or from public or private research centers.
L'archive ouverte pluridisciplinaire HAL, est destinée au dépôt et à la diffusion de documents scientifiques de niveau recherche, publiés ou non, émanant des établissements d'enseignement et de recherche français ou étrangers, des laboratoires publics ou privés. 


\title{
Kinetic Energy Released in the Vibrational Autodetachment of Sulfur Hexafluoride Anion
}

\author{
Bruno Concina ${ }^{1}$, Guillaume Montagne, Serge Martin, Christian Bordas
}

\author{
Institut Lumière Matière, Université de Lyon, Université Claude Bernard Lyon 1, CNRS, \\ F-69622 Villeurbanne, France
}

\begin{abstract}
The kinetic energy release distribution (KERD) in the vibrational autodetachment (VAD) from sulfur hexafluoride anion $\mathrm{SF}_{6}{ }^{-}$has been measured in a velocity map imaging spectrometer for delays in the range of a few tens of microseconds. The experimental KERD is analyzed within the framework of the detailed-balance: first using the standard Langevin model, subsequently using a more refined and realistic model based on the experimental attachment cross-section. A discussion on the processes involved in the attachment and the VAD is presented based on an empirical fit of the attachment cross section. The lifetime derived from the model is in good agreement with the experimental time window, strengthening this theoretical approach for this model system.
\end{abstract}

\section{Introduction}

Sulfur hexafluoride $\left(\mathrm{SF}_{6}\right)$ is of particular interest for industry: it is widely used as a gaseous dielectric and in plasma etching. ${ }^{1-3}$ This molecule is also known to be a greenhouse gas with an extremely long lifetime in the Earth's atmosphere, which reinforces the interest of studying the formation and the decay of the $\mathrm{SF}_{6}{ }^{-}$anion. ${ }^{4-6}$ Non-dissociative electron attachment to $\mathrm{SF}_{6}$ at low energy is characterized by an exceptionally large cross section. ${ }^{7}$ It proceeds by s-wave electron capture and forms mostly longlived $\mathrm{SF}_{6}{ }^{-}$anions by non-dissociative attachment. ${ }^{7}$ Vibrational autodetachment (VAD) from $\mathrm{SF}_{6}{ }^{-}$is a statistical process and is closely related to the (non-dissociative) electron attachment to $\mathrm{SF}_{6}$ through the detailed-balance principle. ${ }^{8}$ Detailed theoretical studies have been devoted to both processes, $\mathrm{SF}_{6}$ being considered as a model system. ${ }^{8-10}$

An accurate description of the attachment and detachment processes requires an accurate determination of the adiabatic electron affinity (AEA) of $\mathrm{SF}_{6}$. The latter has been the subject of a considerable theoretical and experimental work over the years. ${ }^{11,12}$ On the experimental side, the direct AEA measurement is not possible. Indeed, the 0-0 line is invisible because of the poor FranckCondon overlap due to the significant geometry change between the anion and the neutral. ${ }^{13}$ On the

\footnotetext{
${ }^{1}$ Corresponding author : bruno.concina@univ-lyon1.fr
} 
theoretical side, the anion was predicted to have the same $O_{h}$ symmetry as the neutral, the S-F bond being elongated by about $0.2 \AA .{ }^{14}$ However, recent coupled-cluster calculations led to a $C_{4 v}$ distorted geometry for the anion ${ }^{15}$ and an AEA of $1.0340+/-0.03 \mathrm{eV}$ after corrections. ${ }^{16,17}$ Electron affinity may be determined from a third-law analysis of the equilibrium constant $K_{c}$ using experimental data for thermal attachment/detachment and, among others, the vibrational partition function. ${ }^{18}$ The new geometry of $\mathrm{SF}_{6}{ }^{-}$considerably modifies the latter and inspires a reanalysis ${ }^{19}$ leading to $\mathrm{EA}=1.03+/-0.05$ $\mathrm{eV}$, in agreement with the corrected coupled-cluster results. In the following, this value will be considered as the most accurate to date.

$\mathrm{SF}_{6}{ }^{-}$lifetime was investigated in an electrostatic storage ring showing neutralization of hot $\mathrm{SF}_{6}{ }^{-}$by VAD on the microsecond to the millisecond timescale. ${ }^{20}$ The decay exhibits a power law dependence due to the broad distribution of vibrational energy. A cryogenic ion-beam trap with extremely low residual gas densities provided observation of the neutralization rates due to VAD over times up to $100 \mathrm{~ms}^{21}$ The statistical modeling of the experimental decay relies on the accurate description of the attachment process and of the vibrational level densities. In this frame, the distorted $C_{4 V}$ geometry of the anion is essential for achieving a consistent description of the experimental data. The deduced EA value is smaller, but still consistent with the one determined from third-law analysis. ${ }^{19}$

In the present work, we report on the first measurement of the Kinetic Energy Release Distribution (KERD) of $\mathrm{VAD}$ of $\mathrm{SF}_{6}{ }^{-}$. It has been achieved on the timescale of a few tens of microseconds. When compared to the neutralization rate, the KERD brings additional information and allows a more stringent test of the VAD statistical modeling. In the frame of the detailed balance formalism, a crucial point is the choice of the cross section of the reverse process, i.e. the electron attachment to the neutral. The anion formation may be pictured as a two-step process: electron capture in the polarization potential of the neutral followed by the transfer of the electron excess energy to the vibrational modes leading to a stable anionic state, the latter process being called Intramolecular Vibrational Redistribution (IVR). The classical Langevin model provides an estimate of the capture cross section at the most basic level of approximation. ${ }^{22}$ As a classical model, it is not well suited to the description of the s-wave capture to $\mathrm{SF}_{6}$. Besides, it does not deal with the IVR process. A more realistic detailed-balance modeling of VAD is proposed, based on the fit of the experimental attachment cross section ${ }^{7}$ by an empirical expression ${ }^{10}$ including s-wave electron capture and IVR. The experimental KERD is fitted with the one derived from the model, using a single parameter, the microcanonical daughter $\left(\mathrm{SF}_{6}\right)$ temperature. ${ }^{23,24}$ The vibrational energy of the parent $\mathrm{SF}_{6}{ }^{-}$is deduced and used to calculate the electron emission rate, which is finally found in good agreement with the experimental timescale.

This work is part of a long term study on the delayed electron emission of negatively charged molecules and small clusters, interpreted as thermionic emission. Our approach is based on the measurement in a velocity map imaging spectrometer of the delayed electron KERD after photoexcitation and on the development of statistical modeling relying on the detailed-balance principle. Measurements on small negatively charged tungsten clusters showed deviations from the Langevin classical capture model. ${ }^{22}$ The KERD was observed sharper than expected. When compared to the Langevin model, the attachment cross section magnitude deduced from the experiment was found lower by up to two orders of magnitude. ${ }^{22}$ Measured KERD of fullerene anions showed significant variations as a function of the molecular size. ${ }^{25}$ The quantum Vogt and Wannier's formalism was introduced to describe the electron capture and allowed to write an empirical expression for the IVR. Information on the IVR were deduced from the fit of the experimental spectra and were interpreted using the molecular symmetries. ${ }^{25}$ A thermal tunneling detachment was evidenced in the study of the fullerene dianions. ${ }^{24,26} \mathrm{SF}_{6}{ }^{-}$allows a much more stringent test of the thermionic emission modeling than the 
tungsten clusters ${ }^{22}$ or the fullerene anions. ${ }^{25}$ Indeed, there is little uncertainty on the attachment cross section of this model system, which has been measured with high resolution. ${ }^{7}$

The article is organized as follows. In Sec. II, the experimental set-up is briefly described and the experimental KERD is compared to the Langevin model. Sec III is devoted to the presentation of the detailed balance model using an experimental attachment cross section. In Sec. IV, the experimental KERD is compared to model outputs, allowing a discussion on the physical processes. Sec V. shows that the VAD rate derived from the model agrees with the experimental timescale. Finally, Sec. VI is devoted to the conclusion.

\section{Experimental and comparison to the Langevin model}

Hot $\mathrm{SF}_{6}{ }^{-}$anions are produced in an Electron Cyclotron Resonance (ECR) ion source by electron attachment to the $\mathrm{SF}_{6}$ gas. ${ }^{27,28} \mathrm{~A}$ continuous beam of negative ions (and electrons) is extracted from the plasma by an acceleration voltage of a few $\mathrm{kV}$ and is mass analyzed by a $90^{\circ}$ magnetic sector. The mass selected anion beam is pulsed using a parallel plate deflector leading to ion bunches of a few $\mu \mathrm{s}$ duration at $1-\mathrm{kHz}$ rate. The ion bunches pass through a drift tube and enter a velocity map imaging (VMI) spectrometer orthogonally mounted to the ion drift tube. ${ }^{29}$ An inhomogeneous static electric field perpendicular to both the ion trajectory and the laser beam path accelerates the electrons towards a position-sensitive detector (PSD) composed by a microchannel plate detector and a phosphor screen. The voltages applied to the PSD are gated in order to reduce the background. Electron impacts are recorded by a CCD camera. The angularly resolved velocity distribution is calculated from the raw image by numerical inversion. ${ }^{30}$ The KERD is deduced after angular integration. The VMI spectrometer is calibrated with the photoelectron spectrum of $\mathrm{F}^{-}$at $349 \mathrm{~nm}$ laser wavelength. ${ }^{31}$

Unlike the previous studied systems (tungsten clusters ${ }^{22}$ and fullerene anions ${ }^{25}$ ), photoexcitation in the NIR-UV-VIS does not lead to thermionic emission (measurements have been attempted at 1064, 532 and $349 \mathrm{~nm}$ laser wavelength). Only the direct detachment is detected and will be separately discussed in a forthcoming article. Nevertheless, a spontaneous electron emission is visible and can be measured without laser excitation. This decay is due to the internal energy that the $\mathrm{SF}_{6}{ }^{-}$anion has acquired during its production in the ECR source. It is expected that a wide range of internal energy is populated in the ion source, producing a set of excited species whose lifetime covers a broad range. The spontaneous electron emission should depend slightly on the time of flight from the ion extraction to the VMI spectrometer, since hotter species are expected to decay faster. Two acceleration voltages (4 and 6 $\mathrm{kV}$ ) have been used leading to two slightly different times of flight (48 and $39 \mu \mathrm{s})$. No significant differences have been noticed in the measured KERD, certainly because of a too small change in time of flight. The electron raw image and the electron KERD of the vibrational autodetachment of sulfur hexafluoride anions are reported in Fig. 1. The electron distribution on the PSD is isotropic in agreement with the picture of a statistical electron emission.

The geometry of our VMI spectrometer is such that, at least for the low kinetic energies we are currently interested in, all the electrons emitted in the VMI collection zone fulfill the correct operating conditions of the VMI, which allows us to reconstruct with a satisfactory resolution the KERD of the emitted electrons, as verified by two experimental procedures. First, the settings must lead to an isotropic image, which is the only possible geometry when there is no laser beam. Second, it has been checked that the KERD does not change when all VMI voltages are rescaled by the same factor, which changes the size of the image while preserving the spatial focusing conditions. Additionally, the VMI 
voltages are very low to prevent from a large deflection of the ions and to measure in good conditions the very low energy of this KERD: this distribution peaks at $12 \mathrm{meV}$ (an extremely low value only accessible thanks to specific VMI capabilities) and vanishes completely above $200 \mathrm{meV}$. Using the photodetachment spectrum of $\mathrm{F}^{-}$at $349 \mathrm{~nm}$ laser wavelength, a resolution $\Delta \varepsilon=8 \mathrm{meV}$ (FWHM) is found at $\varepsilon=101 \mathrm{meV}$. The scaling law in $\sqrt{\varepsilon}$ leads to $\Delta \varepsilon=3 \mathrm{meV}$ for $\varepsilon=12 \mathrm{meV}$.

A first approach consists in fitting the experimental KERD with the distribution derived from the Langevin model: ${ }^{22}$

$$
f(\varepsilon) \propto \varepsilon^{1 / 2} e^{-\varepsilon / k_{B} T_{d}}
$$

with $\varepsilon$ the electron kinetic energy and $T_{d}$ the microcanonical daughter $\left(\mathrm{SF}_{6}\right)$ temperature. ${ }^{24}$ The fit reproduces nicely the experimental data. The optimized temperature $T_{d}$ is $319 \mathrm{~K}$. An empirical extension of this fit function is frequently used:

$$
f(\varepsilon) \propto \varepsilon^{\gamma} e^{-\varepsilon / k_{B} T_{d}}
$$

The agreement with the experimental curve is slightly improved and is obtained for the parameters $\gamma=0.44$ and $T_{d}=346 \mathrm{~K}$. The two fit functions reproduce quite well the experimental data but a straightforward question arises: are the fitted temperatures meaningful? The Langevin model is based on the classical capture cross section, which is far from the realistic attachment scenario, composed of the s-wave capture and the IVR. As the empirical expression including the adjustable $\gamma$ parameter is not derived from a model, the interpretation of its parameter $T_{d}$ as the daughter temperature is problematical and therefore it should not be regarded as a true temperature. The interest of this type of fitting function is rather to characterize the KERD shape with the $\gamma$ parameter. ${ }^{32}$ Let us point out that our experimental result is at variance with detailed statistical rate calculations combined with electron capture theory and kinetic modeling reported in ref. ${ }^{8}$, which led to an exponent $\gamma \approx 0.15$. All these findings call for a more realistic modeling of $V A D$, as described in the following section.

\section{Detailed-balance modeling of VAD using an experimental attachment cross section}

The vibrational autodetachment (VAD) of the $\mathrm{SF}_{6}{ }^{-}$anion

$$
S F_{6}^{-} \rightarrow S F_{6}+e^{-}
$$

may be studied in the frame of detailed balance. ${ }^{33}$ As the anion and the neutral are both assumed to be in the electronic ground state, the electron binding energy $E_{b}$ is equal to the electron affinity $E A$ of $\mathrm{SF}_{6}(E A=1.03 \mathrm{eV}) \cdot{ }^{19}$ The differential rate for emission of an electron of kinetic energy $\varepsilon$ from a sulphur hexafluoride anion of vibrational energy $E$ reads: ${ }^{24}$

$$
k(E, \varepsilon)=\frac{m_{e}}{\pi^{2} \hbar^{3}} \frac{g_{0}}{g_{-}} \varepsilon \sigma(\varepsilon) \frac{\rho_{0}\left(E-E_{b}-\varepsilon\right)}{\rho_{-}(E)}
$$

with $m_{e}$ the electron mass, $g_{-}$the electronic degeneracy of anion and $g_{0}$ the electronic degeneracy of the neutral times the spin degeneracy of the free electron. $\rho_{0}$ and $\rho_{-}$denote the vibrational density of states respectively for the neutral and the anion. $\sigma$ is the cross section of the reverse process, i.e. the (non-dissociative) electron attachment to $\mathrm{SF}_{6}$. We introduce the daughter temperature $T_{d}$, defined as the microcanonical temperature of the neutral $\mathrm{SF}_{6}$ with vibrational energy $E-E_{b} \cdot{ }^{34}$

$$
\frac{1}{k_{B} T_{d}}=\frac{d}{d E} \ln \rho_{d}\left(E-E_{b}\right)
$$


$\ln \left(\rho_{0}\left(E-E_{b}-\varepsilon\right)\right)$ may be expanded through a Taylor expansion in powers of $\varepsilon$ using the daughter temperature $T_{d} \cdot{ }^{24}$ The first term leads to an Arrhenius factor. The expression of the second and the third terms are given in the Appendix I and depends on the heat capacity $C_{d}$ (temperature derivative of the energy) of the neutral (daughter) $\mathrm{SF}_{6}$ defined for $T=T_{d}$. Finally, the differential rate may be written:

$$
k(E, \varepsilon)=\frac{m_{e}}{\pi^{2} \hbar^{3}} \frac{g_{0}}{g_{-}} \varepsilon \sigma(\varepsilon) \frac{\rho_{0}\left(E-E_{b}\right)}{\rho_{-}(E)} \exp \left(-\frac{\varepsilon}{k_{B} T_{d}}-\cdots\right)
$$

with the dotted line denoting the second and the third terms. In the case the level densities of the neutral and the anion can be assumed to be equal, their ratio may be replaced by an exponential term depending on the so-called emission temperature $T_{e} \cdot{ }^{23}$ In the case of $\mathrm{SF}_{6}$, they differ so much (due to the change of geometry) ${ }^{15}$ that the level densities are kept in place 8,35 and are calculated (in the harmonic approximation) by the Whitten-Rabinovitch expression ${ }^{8,36,37}$ as a function of the internal energy (see the Appendix II). An analytical expression is derived for the caloric curve (i.e. the vibrational energy as a function of the microcanonical temperature, see eq. (A.8)) and thus the heat capacity $C_{d}$ is also calculated, as needed for the second and the third terms of the expansion in powers of $\varepsilon$. Finally, the kinetic energy distribution reads in this theoretical approach:

$$
f(\varepsilon) \propto \varepsilon \sigma(\varepsilon) \exp \left(-\frac{\varepsilon}{k_{B} T_{d}}-\cdots\right)
$$

The full expression of the KERD is available in Appendix I, eq. A.1. The KERD depends on two essential ingredients: the cross section $\sigma(\varepsilon)$ of the reverse process and the daughter temperature $T_{d}$ appearing in the exponential factor. In the case of a KERD with low values of $\frac{\varepsilon}{k_{B} T_{d}}$, the exponential factor has a simple expression: it contains only the first term and the knowledge of the heat capacity $C_{d}$ is not required. If the KERD is characterized by higher values of $\frac{\varepsilon}{k_{B} T_{d}}$, it is necessary to take into account the higher-order terms, which requires the knowledge of $C_{d}$ (see Appendix I).

Additional modeling is needed for expressing the attachment cross section $\sigma(\varepsilon)$. The anion formation may be pictured as a two-step process: electron capture in the polarization potential followed by transfer of the electron excess energy to the vibrations leading to a stable anionic state. The quantum description of the first step was developed by E. Vogt and G. H. Wannier. ${ }^{38}$ The capture cross section is the sum of the contributions of the partial waves of given orbital momentum $\ell$ and reads in atomic units as a function of the reduced wave number $\kappa:^{25}$

$$
\sigma(\kappa)=\alpha \frac{\pi}{\kappa^{2}} \sum_{\ell}(2 \ell+1) P_{\ell}^{V W}(\kappa)
$$

with $P_{\ell}^{V W}(\kappa)$ the orbital momentum-dependent capture probability and $\alpha$ the polarizability of $\mathrm{SF}_{6}(\alpha=$ $\left.44.1 a_{0}^{3}\right) .{ }^{39}$ The reduced wave number $\kappa$ reads in atomic units : $\kappa=\sqrt{2 \alpha \varepsilon}$. In the case of $\mathrm{SF}_{6}$, the attachment proceeds only by s-wave capture: ${ }^{7}$

$$
\sigma(\kappa)=\alpha \frac{\pi}{\kappa^{2}} P_{0}^{V W}(\kappa)
$$

A simple and accurate analytical expression is available for $P_{0}^{V W}(\kappa):^{40}$

$$
P_{0}^{V W}(\kappa)=1-0.25 e^{-1.41 \kappa}-0.75 e^{-4.86 \kappa}
$$

The cross section of the non-dissociative electron attachment to $\mathrm{SF}_{6}$ was measured by Braun et al. with high resolution on a large electron energy range (0.1-500 meV). ${ }^{7}$ It is represented as a function of $\kappa$ in 
Fig. 2. It allows to determine empirically the second step of electron attachment. In this simple picture, the non-dissociative attachment cross section reads: ${ }^{9,10}$

$$
\sigma(\kappa)=\alpha \frac{\pi}{\kappa^{2}} P_{0}^{V W}(\kappa) P^{I V R}(\kappa) P^{V E X}(\kappa)
$$

The factor $P^{I V R}(\kappa)$ characterizes the intramolecular vibrational redistribution (IVR), i.e. the transfer of some electronic energy to the vibrations to lead to a stable anion; this factor may be given by the empirical expression: ${ }^{9,10}$

$$
P^{I V R}(\kappa)=e^{-c_{1} \kappa^{2}}
$$

The parameter $c_{1}$ is deduced from the fit with the experimental cross section. Additionally, some electron captures may lead to vibrational excitation (VEX) and then electron release. The energy threshold for such an inelastic collision is given by the quantum of the symmetric stretch mode $A_{1 g}$ (96.6 meV) ${ }^{7}$ leading to $\kappa_{0}=0.560$. The factor $P^{V E X}(\kappa)$ may be written as:

$$
\begin{array}{cc}
P^{V E X}(\kappa)=1 & \text { for } \kappa \leq \kappa_{0} \\
P^{V E X}(\kappa)=e^{-c_{2}\left(\kappa^{5}-\kappa_{0}^{5}\right)} & \text { for } \kappa \geq \kappa_{0}
\end{array}
$$

Finally, the two free parameters $c_{1}$ and $c_{2}$ are deduced from the fit to the experimental cross section: $c_{1}=2.5$ and $c_{2}=3.4$ (see Fig. 2).

One deduces from Eq. (7) the detailed-balance expression of the $\kappa$ distribution: ${ }^{25}$

$$
f(\kappa) \propto \kappa^{3} \sigma(\kappa) \exp \left(-\frac{\kappa^{2}}{2 \alpha k_{B} T_{d}}-\cdots\right)
$$

Finally the $\kappa$ distribution used to fit the experimental one reads:

$$
f(\kappa) \propto \kappa P_{0}^{V W}(\kappa) P_{e l}^{I V R}(\kappa) P^{V E X}(\kappa) \exp \left(-\frac{\kappa^{2}}{2 \alpha k_{B} T_{d}}-\cdots\right)
$$

It may be noticed that, in the factor $\frac{\kappa^{2}}{2 \alpha k_{B} T_{d}}, \alpha$ is given in atomic units and $T_{d}$ in Kelvin, leading to the Boltzmann constant $k_{B}$ in Hartree by Kelvin : $k_{B}=3.1668 \times 10^{-6} \mathrm{HK}^{-1} .{ }^{25}$

\section{Discussion on the reduced-wave-number $\boldsymbol{\kappa}$ distribution of VAD}

The experimental $k$ distribution is reported in Fig. 3. It is compared with several fits respectively based on different assumptions regarding the attachment cross section. In all cases, the fit expressions include the second order term in $\varepsilon$. A large variation of the daughter temperature $T_{d}$ may be observed whereas in all cases the agreement with the experimental curve is quite satisfactory. The small differences between the three fit curves occur in the high $\kappa$ region where the signal is quite low and consequently may not be significant. Assuming a pure s-wave capture leads to a daughter temperature $T_{d}$ equal to $479 \mathrm{~K}$. It is significantly higher than the temperature deduced from the Langevin capture cross section $\left(T_{d}=319 \mathrm{~K}\right.$ ). The temperature increases when the cross section is reduced by assuming a second step in the attachment process: IVR alone leading to $T_{d}=652 \mathrm{~K}$, or IVR and VEX leading to $T_{d}=$ $667 \mathrm{~K}$. The influence of the IVR is dominant in this increase. Only a change of $2 \%$ is induced by VEX.

As mentioned above, the expansion of the density of state has been cut at the second order. The position of the cut has been determined by calculating the fits at different orders assuming s-wave capture followed by IVR and VEX. A fit with only the first order term leads to $T_{d}=620 \mathrm{~K}$, showing a 
significant difference $(7 \%)$ with the second order result $\left(T_{d}=667 \mathrm{~K}\right)$. By contrast, including the third order term changes by less than $1 \%$ the daughter temperature $\left(T_{d}=672 \mathrm{~K}\right)$, which justifies to cut the expansion at the second order. Finally, as enough terms are given in the expansion and a realistic model is used to describe the reverse process, the temperature $T_{d}=667 \mathrm{~K}$ is meaningful.

As outlined in ref. ${ }^{8}$, detailed balance should link truly reverse processes between, on one side, the anion in the electronic ground state and, on the other side, the neutral and the electron infinitely separated. The competition with the vibrational excitation (VEX) has to be included in the attachment cross section: first the electron is captured and then the IVR takes place whereas VEX does not occur. But the detachment is presumably not concerned with VEX: the inverse process of IVR takes place and then the inverse process of electron capture occurs. Finally when comparing with experiment, this point is of little concern: most of the $\kappa$ distribution is defined below the VEX threshold $\left(\kappa_{0}=0.560\right.$, materialized by a red arrow on Fig. 3), which explains why VEX has a so little influence on the temperature. Besides, above the VEX threshold, the fit of the attachment cross section allows only the determination of the product $P^{I V R}(\kappa) P^{V E X}(\kappa)$. Another expressions for both $P^{I V R}(\kappa)$ and $P^{V E X}(\kappa)$ might be assumed as much as the product remains the same. This uncertainty on the empirical factors prevents from an exact determination of the cross section employed in the detailed-balance expression of VAD.

In order to reinforce our approach, the VAD rate derived from the model is compared with the experimental lifetime in the next section.

\section{Discussion on the VAD rate}

As outlined in section II, the internal energy distribution of the $\mathrm{SF}_{6}{ }_{6}$ anion is broad. As a consequence, the time dependence of the decay rate follows a power law (and not an exponential as expected for a very narrow energy distribution). ${ }^{34,41}$ Nevertheless, the internal energy distribution of the anions decaying at a given time $t$ is relatively sharp and peaks at the energy $\bar{E}$ given by $K(\bar{E})=1 / t$ where $K(\bar{E})$ is the rate for electron emission from an anion of internal energy $\bar{E} \cdot{ }^{24,34,41}$ In the following, the time $t$ stands for the time of flight from the ECR source to the VMI spectrometer ( 48 or $39 \mu$ s ; average value : $44 \mu \mathrm{s})$. The model would be reinforced if agreement was found between the experimental time of flight $t$ and the inverse $\tau(E)$ of the theoretical emission rate $K(E)$ with $E$ the internal energy of the anion deduced from the experimental KERD via the temperature $T_{d}=667 \mathrm{~K}$ of the neutral.

Using the caloric curve of the neutral, $T_{d}=667 \mathrm{~K}$ leads to $E-E_{b}=0.36 \mathrm{eV}$ and $E=1.39 \mathrm{eV}$ ( $E A=$ $1.03 \mathrm{eV}$ from Ref. $\left.{ }^{19}\right)$. For such an internal energy, radiative cooling can be disregarded: it has been shown to compete with VAD only if $E-E_{b} \lesssim 50-100 \mathrm{meV} .{ }^{21}$ By integrating Eq. (6), one deduces the rate for electron emission from a sulphur hexafluoride anion of vibrational energy $E$ :

$$
K(E)=\frac{m_{e}}{\pi^{2} \hbar^{3}} \frac{g_{0}}{g_{-}} \frac{\rho_{0}\left(E-E_{b}\right)}{\rho_{-}(E)} \int_{0}^{E-E_{b}} \varepsilon \sigma(\varepsilon) \exp \left(-\frac{\varepsilon}{k_{B} T_{d}}-\cdots\right) d \varepsilon
$$

Rate calculations are carried out to the second order in $\varepsilon$ so as to be consistent with the $\kappa$ distribution fits. As defined above, $g_{0}$ is the electronic degeneracy of the neutral times the spin degeneracy of the free electron and $g_{-}$is the electronic degeneracy of the anion. Since all molecular orbitals of $\mathrm{SF}_{6}$ are full, ${ }^{11} g_{0}$ and $g_{-}$are both equal to 2 . As mentioned previously the densities of state and the caloric curve are calculated in the harmonic approximation using the Whitten-Rabinovitch expression (see eq. A.3 and A.8 of Appendix II). Using the vibrational frequencies of $\mathrm{O}_{\mathrm{h}}$ symmetry, ${ }^{14}$ one finds for $\mathrm{SF}_{6}$ : 


$$
\rho_{0}\left(E-E_{b}\right)=23 / \mathrm{cm}^{-1}
$$

In a first approach, $\mathrm{O}_{h}$ symmetry is assumed for $\mathrm{SF}_{6}$. Using vibrational frequencies from Ref. ${ }^{14}$, one gets:

$$
\rho_{-}(E)=2.5 \cdot 10^{8} / \mathrm{cm}^{-1}
$$

leading to a rate $K(E)=5.2 \cdot 10^{5} \mathrm{~s}^{-1}$ (with $E=1.39 \mathrm{eV}$ ). A lifetime is defined as the inverse of the rate: : $\tau(E) \sim 2 \mu s$. It would mean that, following the emission rate of the model, the internal energy distribution of the molecular anions decaying after a delay $\tau(E) \sim 2 \mu \mathrm{s}$ would peak at the internal energy $E=1.39 \mathrm{eV} \cdot{ }^{24,34,41}$ Consequently, the inverse of the rate $(\sim 2 \mu \mathrm{s})$ may be compared with the ion time of flight between the ECR source and the detection zone $(\sim 44 \mu \mathrm{s})$. It is found one order of magnitude lower than the experimental delay. However, as mentioned earlier, recent calculations demonstrate a $C_{4 v}$ distorted geometry for $\mathrm{SF}_{6}{ }^{-15}$ which leads to a density of states one order of magnitude larger:

$$
\rho_{-}(E)=2.2 \cdot 10^{9} / \mathrm{cm}^{-1}
$$

In this case, the rate is given by $K(E)=5.8 \cdot 10^{4} \mathrm{~s}^{-1}$ and the lifetime amounts to $\tau \sim 17 \mu \mathrm{s}$, improving significantly the agreement with the experiment. The distortion of the anion from $O_{h}$ geometry results in considerable anharmonicity, ${ }^{15,19}$ that should significantly increase the density of states $\rho_{-}(E)$ and the lifetime $\tau$, leading certainly to a better agreement with the experimental time of flight. However precise data and spectroscopic constants for describing the anharmonicity are not available. The simple harmonic calculation we have developed already gives a satisfactory agreement with the experiment.

\section{Conclusion}

The KERD of VAD from sulfur hexafluoride anion $\mathrm{SF}_{6}{ }^{-}$has been measured for the first time. It has been compared to outputs from two detailed balance models: Langevin model and a more sophisticated modeling based on a measured attachment cross section. The latter considers a relatively realistic scenario for the attachment: s-wave electron capture followed by IVR and including competition with VEX. The temperature derived from the fitting of the experimental KERD significantly depends on the considered processes. It is also necessary to take into account the second order term in the expansion of the density of states as a function of $\varepsilon$. The temperature derived from the Langevin model is significantly lower than the ones from the more realistic model. Langevin model is not well suited for the VAD from $\mathrm{SF}_{6}{ }^{-}$: it describes the attachment as a classical capture which is far from the s-wave capture observed for $\mathrm{SF}_{6}{ }^{-}$and it does not deal with IVR. Besides, it should also include higher order terms in power of $\varepsilon$.

VAD rate derived from the realistic model gives lifetime slightly lower (by a factor 2-3) than the experimental time window, which is considered as encouraging for calculations based on harmonic densities of states. It is expected that the $C_{4 v}$ distorted geometry of $\mathrm{SF}_{6}-$ results in considerable anharmonicity, ${ }^{15,19}$ which should increase significantly the calculated lifetime.

The electron attachment to the model molecule $\mathrm{SF}_{6}$ has been well characterized thanks to a large number of studies. ${ }^{7,9,10}$ Thus, the influence of the different processes involved in the attachment can be determined on the VAD rate. It has been shown in this work that the temperature derived from a fit of the KERD is meaningful only if the attachment cross section used in the fit function is realistic and enough terms are included in the expansion as a function of powers of $\varepsilon$. 


\section{Appendix I: Taylor expansion of $\ln \left(\rho_{0}\left(E-E_{b}-\varepsilon\right)\right)$}

$\ln \left(\rho_{0}\left(E-E_{b}-\varepsilon\right)\right)$ may be expanded through a Taylor expansion in powers of $\varepsilon$ using the daughter temperature $T_{d}{ }^{24}$ The first term leads to an Arrhenius factor. The expression of the second and the third terms depends on the heat capacity $C_{d}$ (temperature derivative of the energy) of the neutral (daughter) $\mathrm{SF}_{6}$ defined for $T=T_{d}$. Finally, the kinetic energy distribution reads:

$$
f(\varepsilon) \propto \varepsilon \sigma(\varepsilon) \exp \left(-\frac{\varepsilon}{k_{B} T_{d}}-\frac{1}{2} \frac{k_{B}}{C_{d}}\left(\frac{\varepsilon}{k_{B} T_{d}}\right)^{2}-\frac{1}{6}\left(\frac{k_{B}}{C_{d}}\right)^{2}\left(\frac{\varepsilon}{k_{B} T_{d}}\right)^{3}\left(2+T_{d} \frac{d C_{d}}{d E}\right)\right)
$$

Using the reduced wave number $\kappa=\sqrt{2 \alpha \varepsilon}$ (atomic unit expression), the distribution becomes :

$$
f(\kappa) \propto \kappa^{3} \sigma(\kappa) \exp \left(-\frac{\kappa^{2}}{2 \alpha k_{B} T_{d}}-\frac{1}{2} \frac{k_{B}}{C_{d}}\left(\frac{\kappa^{2}}{2 \alpha k_{B} T_{d}}\right)^{2}-\frac{1}{6}\left(\frac{k_{B}}{C_{d}}\right)^{2}\left(\frac{\kappa^{2}}{2 \alpha k_{B} T_{d}}\right)^{3}\left(2+T_{d} \frac{d C_{d}}{d E}\right)\right)
$$

\section{Appendix II: the Whitten-Rabinovitch approximation}

The harmonic vibrational density of states of $\mathrm{SF}_{6}$ and $\mathrm{SF}_{6}{ }^{-}$may be conveniently calculated in the frame of the Whitten-Rabinovitch approximation: $8,36,37$

$$
\rho(E)=\frac{\left(E+a(E) E_{z}\right)^{s-1}}{(s-1) ! \prod_{i=1}^{S} h v_{i}}
$$

with $h v_{i}$ the $s$ vibrational quanta and $E_{z}=\frac{1}{2} \sum_{i=1}^{s} h v_{i}$ the vibrational zero point energy. The approximation relies on the correction function $a(E)=1-\beta \omega$ which depends on

$$
\begin{array}{ll}
\omega^{-1}=5 \frac{E}{E_{z}}+2.73 \sqrt{\frac{E}{E_{z}}}+3.51 & \text { for } E<E_{z} \\
\log _{10} \omega=-1.0506\left(\frac{E}{E_{z}}\right)^{1 / 4} & \text { for } E>E_{z}
\end{array}
$$

and

$$
\beta=\frac{s-1}{s} \frac{\overline{v^{2}}}{\bar{v}^{2}}=(s-1) \frac{\sum_{i=1}^{s} v_{i}^{2}}{\left(\sum_{i=1}^{s} v_{i}\right)^{2}}
$$

These calculations employ the vibrational frequencies respectively from Ref. ${ }^{14}$ for both $\mathrm{SF}_{6}$ and $\mathrm{SF}_{6}{ }^{-}$of $\mathrm{O}_{h}$ symmetry and from Ref. ${ }^{15}$ for $\mathrm{SF}_{6}{ }^{-}$of $C_{4 v}$ symmetry. Using the definition of the microcanonical temperature

$$
\frac{1}{k_{B} T}=\frac{d}{d E} \ln \rho(E)
$$

an analytical relation between the temperature $T$ and the vibrational energy $E$ may be calculated:

with

$$
(s-1) k_{B} T=\frac{E+(1-\beta \omega) E_{z}}{1-\beta \frac{d \omega}{d E} E_{z}}
$$




$$
\begin{array}{cc}
\frac{d \omega}{d E}=-\omega^{2}\left(\frac{5}{E_{z}}+\frac{2.73}{2 \sqrt{E E_{z}}}\right) & \text { for } E<E_{z} \\
\frac{d \omega}{d E}=-\omega \frac{1.056 \ln 10}{4} \frac{1}{E_{z}^{1 / 4} E^{3 / 4}} & \text { for } E>E_{z}
\end{array}
$$

\section{Data availability}

The data that support the findings of this study are available from the corresponding author upon reasonable request.

\section{References}

${ }^{1}$ L.E. Kline, D.K. Davies, C.L. Chen, and P.J. Chantry, Journal of Applied Physics 50, 6789 (1979).

${ }^{2}$ S. Jesse, A.J. Pedraza, J.D. Fowlkes, and J.D. Budai, J. Mater. Res. 17, 1002 (2002).

${ }^{3}$ L.G. Christophorou and J.K. Olthoff, Fundamental Electron Interactions with Plasma Processing Gases (Springer US, Boston, MA, 2004).

${ }^{4}$ M. Maiss and C.A.M. Brenninkmeijer, Environ. Sci. Technol. 32, 3077 (1998).

${ }^{5}$ R.A. Morris, T.M. Miller, A.A. Viggiano, J.F. Paulson, S. Solomon, and G. Reid, J. Geophys. Res. 100, 1287 (1995).

${ }^{6}$ T. Reddmann, R. Ruhnke, and W. Kouker, J. Geophys. Res. 106, 14525 (2001).

${ }^{7}$ M. Braun, S. Marienfeld, M.-W. Ruf, and H. Hotop, J. Phys. B: At. Mol. Opt. Phys. 42, 125202 (2009).

${ }^{8}$ J. Troe, T.M. Miller, and A.A. Viggiano, The Journal of Chemical Physics 130, 244303 (2009).

9 J. Troe, T.M. Miller, and A.A. Viggiano, The Journal of Chemical Physics 127, 244303 (2007).

${ }^{10}$ H.-J. Troe, G. Marowsky, N.S. Shuman, T.M. Miller, and A.A. Viggiano, Zeitschrift Für Physikalische Chemie 225, 1405 (2011).

${ }^{11}$ L.G. Christophorou and J.K. Olthoff, Journal of Physical and Chemical Reference Data 29, 267 (2000).

${ }^{12}$ N.R. Brinkmann and H.F. Schaefer III, Chemical Physics Letters 381, 123 (2003).

${ }^{13}$ J.C. Bopp, J.R. Roscioli, M.A. Johnson, T.M. Miller, A.A. Viggiano, S.M. Villano, S.W. Wren, and W.C. Lineberger, J. Phys. Chem. A 111, 1214 (2007).

${ }^{14}$ G.L. Gutsev and R.J. Bartlett, Molecular Physics 94, 121 (1998).

${ }^{15}$ W. Eisfeld, The Journal of Chemical Physics 134, 054303 (2011).

${ }^{16}$ W. Eisfeld, The Journal of Chemical Physics 134, 129903 (2011).

${ }^{17}$ A. Karton and J.M.L. Martin, The Journal of Chemical Physics 136, 197101 (2012).

${ }^{18}$ A.A. Viggiano, T.M. Miller, J.F. Friedman, and J. Troe, The Journal of Chemical Physics 127, 244305 (2007).

19 J. Troe, T.M. Miller, and A.A. Viggiano, The Journal of Chemical Physics 136, 121102 (2012).

20 J. Rajput, L. Lammich, and L.H. Andersen, Phys. Rev. Lett. 100, 153001 (2008).

${ }^{21}$ S. Menk, S. Das, K. Blaum, M.W. Froese, M. Lange, M. Mukherjee, R. Repnow, D. Schwalm, R. von Hahn, and A. Wolf, Phys. Rev. A 89, 022502 (2014).

${ }^{22}$ B. Concina, B. Baguenard, F. Calvo, and C. Bordas, The Journal of Chemical Physics 132, 104307 (2010).

${ }^{23}$ J.U. Andersen, E. Bonderup, and K. Hansen, The Journal of Chemical Physics 114, 6518 (2001).

${ }^{24}$ B. Concina, F. Lépine, and C. Bordas, The Journal of Chemical Physics 146, 224311 (2017).

25 B. Concina, F. Lépine, and C. Bordas, Phys. Rev. A 90, 033415 (2014).

${ }^{26}$ B. Concina, F. Lépine, and C. Bordas, Phys. Rev. A 92, 023410 (2015).

${ }^{27}$ S. Martin, J. Bernard, G. Montagne, R. Brédy, B. Concina, and L. Chen, J. Phys.: Conf. Ser. 388, 102011 (2012).

${ }^{28}$ B. Concina, F. Lépine, S. Martin, and C. Bordas, J. Phys.: Conf. Ser. 1412, 242002 (2020). 
${ }^{29}$ B. Concina, E. Papalazarou, M. Barbaire, C. Clavier, J. Maurelli, F. Lépine, and C. Bordas, Rev. Sci. Instrum. 87, 033103 (2016).

${ }^{30}$ C. Bordas, F. Paulig, H. Helm, and D.L. Huestis, Review of Scientific Instruments 67, 2257 (1996).

${ }^{31}$ C. Blondel, C. Delsart, and F. Goldfarb, J. Phys. B: At. Mol. Opt. Phys. 34, L281 (2001).

${ }^{32}$ F. Calvo, F. Lépine, B. Baguenard, F. Pagliarulo, B. Concina, C. Bordas, and P. Parneix, The Journal of Chemical Physics 127, 204312 (2007).

${ }^{33}$ V. Weisskopf, Phys. Rev. 52, 295 (1937).

${ }^{34}$ J.U. Andersen, E. Bonderup, and K. Hansen, J. Phys. B: At. Mol. Opt. Phys. 35, R1 (2002).

${ }^{35}$ L.H. Andersen, Phys. Rev. A 78, 032512 (2008).

${ }^{36}$ G.Z. Whitten and B.S. Rabinovitch, The Journal of Chemical Physics 38, 2466 (1963).

${ }^{37}$ W. Forst, Chem. Rev. 71, 339 (1971).

${ }^{38}$ E. Vogt and G.H. Wannier, Phys. Rev. 95, 1190 (1954).

${ }^{39}$ John R. Rumble, ed., CRC Handbook of Chemistry and Physics, 100th Edition (Internet Version 2019) (CRC Press/Taylor \& Francis, Boca Raton, FL, 2019).

${ }^{40}$ E.I. Dashevskaya, I. Litvin, E.E. Nikitin, and J. Troe, Phys. Chem. Chem. Phys. 11, 9364 (2009).

${ }^{41}$ J.U. Andersen, E. Bonderup, K. Hansen, P. Hvelplund, B. Liu, U.V. Pedersen, and S. Tomita, The European Physical Journal D - Atomic, Molecular and Optical Physics 24, 191 (2003). 


\section{Figure captions}

Figure 1: measured electron KERD of the vibrational autodetachment of $S_{6}^{-}$(full black line), fit based on the Langevin model with the parameter $T_{d}=319 \mathrm{~K}$ (blue dotted line) and empirical fit derived from the Langevin model with the parameters $\gamma=0.44$ and $T_{d}=346 \mathrm{~K}$ (red full line). Inset: electron raw image

Figure 2: measured cross section of the non-dissociative electron attachment to $\mathrm{SF}_{6}$ from Ref. ${ }^{7}$ (black squares), s-wave capture cross section (black line) and fit of the experimental curve including IVR and VEX leading to $c_{1}=2.5$ and $c_{2}=3.4$ (red line).

Figure 3: measured $k$ distribution (black line) compared to fits based on different assumptions concerning the attachment cross section: pure s-wave capture (blue line), s-wave capture followed by IVR (dashed red line), s-wave followed by IVR and VEX (full red line). VEX threshold $\left(\kappa_{0}=0.560\right)$ is pointed by a red arrow. All fit expressions include the second order term in $\varepsilon$. 


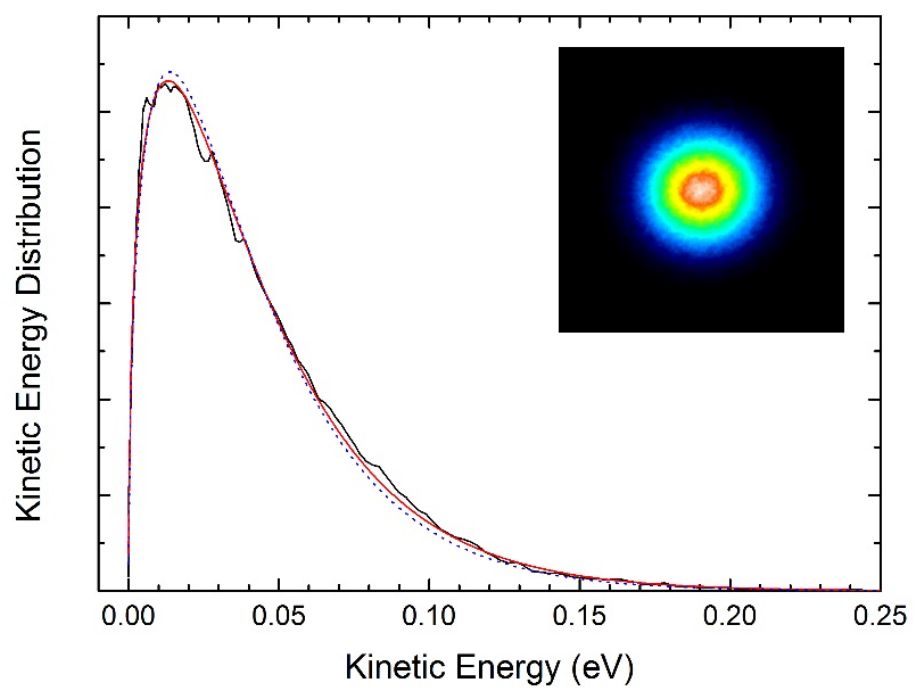

Figure 1 


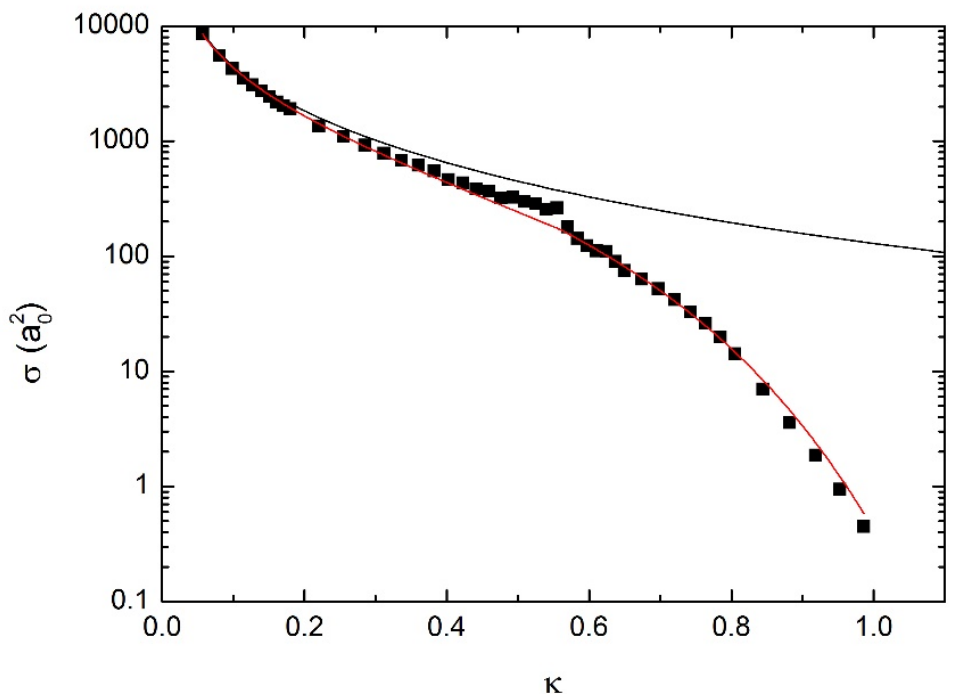

Figure 2 


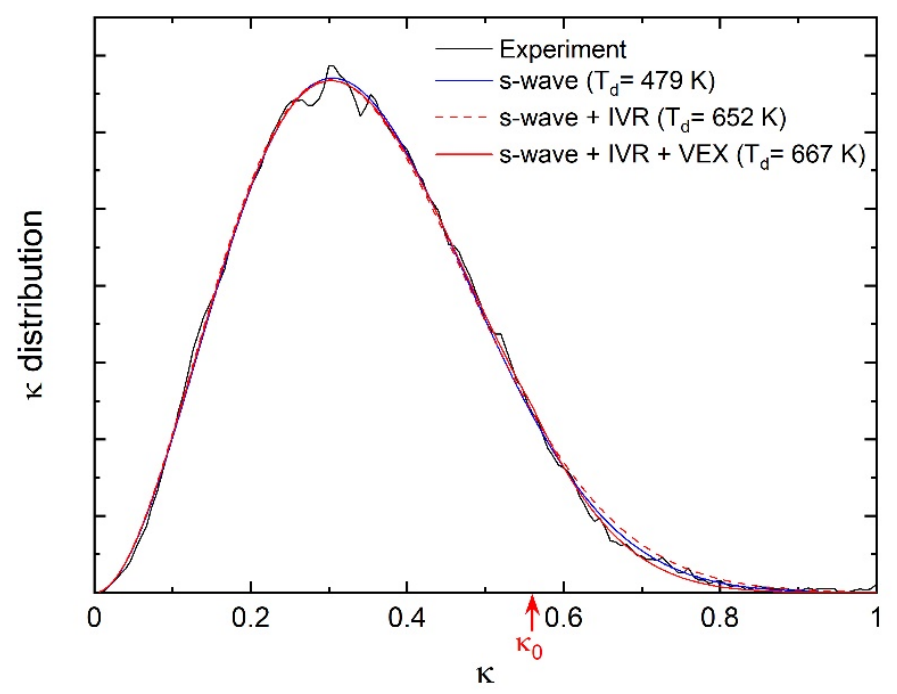

Figure 3 